\title{
REACCIÓN DE VARIEDADES DE FRIJOL COMÚN A Fusarium spp. Y Rhizoctonia solani EN EL ALTIPLANO DE MÉXICO 1
}

\author{
Rosa Navarrete-Maya², Jorge Acosta-Gallegos ${ }^{3}$
}

\begin{abstract}
RESUMEN
Reacción de variedades de frijol común a Fusarium spp. y Rhizoctonia solani en el altiplano de México. En Texcoco, Edo. de México se realizaron dos experimentos, en uno se sembraron bajo riego 32 genotipos de frijol, en abril 24 y mayo 8 de 1997, para evaluar su reacción a Fusarium spp. en un suelo naturalmente infestado. En el otro se evaluó la eficiencia del tratamiento a la semilla con captan y benomyl, para proteger a ocho genotipos del ataque de Fusarium spp. y $R$. solani. En el primero la mayoría de los genotipos resultaron susceptibles a Fusarium spp., sobre todo en la tercera lectura (R6) en ambas fechas de siembra, con excepción de Negro Tacana y Amarillo de Calpan que en la segunda fecha fueron intermedios. La incidencia de Fusarium spp. fue mayor del $80 \%$. En la primera fecha las variedades Pinto Villa y Negro Perla fueron moderadamente resistentes. En el segundo experimento el tratamiento químico favoreció un buen establecimiento de las plantas. Los genotipos Pinto Villa y BAT 477 fueron los menos afectados, tratados con benomyl y captan, respectivamente. Las plantas de genotipos tolerantes sobrevivieron debido al desarrollo de raíces adventicias.
\end{abstract}

\begin{abstract}
Reaction of common bean varieties to Fusarium spp. and Rhizoctonia solani in Mexican Highlands. Two trials were carried out at Texcoco, State of Mexico: in the first one 32 bean genotypes were sowed under irrigation on April 24 and May 8, 1997, in order to study their reaction to Fusarium spp. in a naturally infested soil. In the second one, protection against Fusarium spp. y $R$. solani was evaluated by applying captan or benomyl to the seeds of eight genotypes. In the first trial most genotypes were susceptible to Fusarium spp., particularly in the third evaluation (R6), on both sowing dates; with the exception of Negro Tacana and Amarillo de Calpan, both of which showed an intermediate reaction in the second sowing date. Incidence of Fusarium spp. was higher than $80 \%$. On the first sowing date the Pinto Villa and Negro Perla genotypes were moderately resistant. In the second experiment the chemical treatment produced more vigorous seedlings. Pinto Villa and BAT 477 genotypes showed lower severity scores when treated with benomyl and captan, respectively. It was observed that plants of resistant genotypes survived because they developed adventitious roots.
\end{abstract}

\section{INTRODUCCION}

En el Altiplano Central de México el frijol (Phaseolus vulgaris $\mathrm{L}$.) es un cultivo de subsistencia que se produce con bajo uso de insumos, su rendimiento es afectado por problemas como enfermedades, plagas, sequía y baja fertilidad de los suelos. En las regiones productoras de frijol de temporal, que son las predominantes en el Altiplano, se han detectado diversos patógenos, entre los que destacan los causantes de las pudriciones de la raíz: Fusarium solani (Martius) Appel \& Wr f. sp. phaseoli (Burk) Snyder y Hansen, F. oxysporum Schlecht. f. sp. phaseoli Kendrick \& Snyder y Rhi- zoctonia solani Kühn (Sánchez y Cárdenas, 1988; López, 1991). Estos hongos se han encontrado atacando frijol en 13 estados del país (Campos, 1987; Becerra y López, 1994; Velasquez-Valle y Schwartz, 1997).

Las pudriciones de la raíz inducidas por las especies de Fusarium provocan pérdidas debido a que la infección puede ocurrir desde el momento de la siembra hasta que el frijol es una planta adulta. Los síntomas son más severos cuando las condiciones del suelo son pobres, con drenaje inadecuado, con estructura pobre, bajo contenido de materia orgánica y compactados. Es frecuente observar pudriciones de raíz en siembras de

\footnotetext{
1 Trabajo presentado en la XLIV Reunión anual del PCCMCA, Nicaragua. 1998. Proyecto parcialmente financiado por Bean/Cowpea-CRSP, Title XII, Grant No. USAID DAN-BIO-6-55-6008-00.

2 UNIGRAS, FES-Cuautitlán, UNAM, Cuautitlán Izcalli, Edo. de México, Apdo. Postal 25.

3 CEVAMEX-INIFAP, Texcoco, Edo. de México, Apdo. Postal 10.
} 
riego, como manchones o en forma aislada (Campos, 1987), también es frecuente observarlas cuando no se practica la rotación de cultivos, lo que favorece el incremento en la cantidad de inóculo. La severidad del daño provocado por estos patógenos está influenciada directamente por las condiciones ambientales (sequía y las altas temperaturas) y del suelo, sobre todo en las primeras etapas fenológicas del cultivo (Abawi y PastorCorrales, 1990).

La problemática de la pudrición de raíces se incrementa debido a que las especies de Fusarium, además de dispersarse a través de los aperos de labranza, pueden ser transmitidas por la semilla (Navarrete y Acosta, 1996) y en México, muchos de los productores emplean como semilla el grano que ellos mismos producen para iniciar el nuevo ciclo agrícola (Rodríguez, 1996).

Los síntomas iniciales de la pudrición de raíces por Fusarium solani f. sp. phaseoli son la presencia de estrías longitudinales rojizas en el hipocótilo y en la raíz primaria de las plántulas. Conforme progresa la infección todo el sistema radical puede cubrirse de lesiones café rojizas superficiales, en ocasiones se presentan fisuras y la raíz se hace hueca. Si la raíz primaria muere, la planta se observa marchita, achaparrada y con defoliación prematura (Abawi, 1989). Los síntomas de pudriciones radicales por $F$. oxysporum f. sp. phaseoli pueden confundirse con los de una deficiencia de fósforo, hay amarillamiento y marchitez de las hojas inferiores. Las raíces muestran una coloración café rojiza que puede extenderse al tallo (Abawi, 1989).

En el caso de $R$. solani, las raíces infectadas muestran lesiones ovales a nivel de la corteza de la raíz, cuello e hipocótilo, el tejido se deshidrata y se hunde. La lesión se torna café rojiza y en ocasiones el borde tiene un color más obscuro, las lesiones se pueden unir e incluso llegar hasta el cuello. Las plantas atacadas se muestran marchitas (Abawi y Pastor-Corrales, 1990).

Una de las estrategias que existen para el combate de estas enfermedades es el uso de variedades resistentes (Campos, 1987; Singh, 1992; Velasquez-Valle y Schwartz, 1997). Cuando no se dispone de este tipo de variedades puede recurrirse a otras medidas de control, como el uso de agroquímicos aplicados al suelo o a la semilla, el control cultural mediante la rotación de cultivos, la siembra en bordos levantados, el manejo de las fechas de siembra, y el control biológico mediante el empleo de microorganismos que controlen a los patógenos inductores de las pudriciones de la raíz (Abawi y Pastor-Corrales, 1990; Estevez, et al. , 1998).
Los objetivos del presente trabajo fueron: 1) evaluar la reacción de 32 genotipos de frijol a las pudriciones de raíz inducidas por Fusarium spp. y 2) evaluar la eficiencia del tratamiento químico a la semilla para el control de las pudriciones de la raíz en ocho genotipos de frijol, en un suelo naturalmente infestado por los hongos Fusarium spp. y $R$. solani.

\section{MATERIALES Y MÉTODOS}

\section{Sitio experimental}

Los experimentos se establecieron en el Campo Experimental Valle de México del INIFAP, Texcoco, Edo. de México (19²9' N, 9851' O y 2240 msnm), sitio con clima templado húmedo con inviernos benignos (García, 1981) y con $640 \mathrm{~mm}$ de precipitación anual. El suelo utilizado cuenta con un historial de alta infestación por Fusarium spp. y Rhizoctonia solani, es de tipo migajón arenoso profundo, del orden Molisol (Clasificación FAO-UNESCO), con un epipedon mólico (de color obscuro, rico en humus en los estratos superficiales), generalmente con un $\mathrm{pH}$ neutro y materia orgánica con un contenido de nitrógeno de moderado a alto.

Para verificar la infestación por Fusarium spp. en el suelo, se tomaron al azar muestras de éste y se hicieron aislamientos según el método de Abawi y Pastor-Corrales (1990). Se obtuvo Fusarium spp. en el 100\% de las muestras. Durante el desarrollo del experimento se llevó un registro de la información climática diaria: temperatura, humedad relativa y precipitación, en una estación meteorológica localizada a $500 \mathrm{~m}$ del sitio experimental.

Se llevaron a cabo dos experimentos: 1) evaluar el ataque por Fusarium spp. a 32 genotipos de frijol y 2) evaluar la eficiencia del tratamiento químico a la semilla de ocho genotipos, para el control de Fusarium spp. y $R$. solani.

\section{Experimento 1}

Los genotipos se sembraron en surcos únicos de siete $\mathrm{m}$ de longitud, en cuatro sub-bloques de ocho genotipos con dos repeticiones. Se realizó una aleatorización de genotipos en ambos sub-bloques y repeticiones. Para la conducción del cultivo se realizaron las prácticas culturales recomendadas para el frijol en la región. Se realizaron dos siembras, una el 24 de abril y otra el 8 de mayo de 1997. Para el establecimiento del cultivo se dio un riego de presiembra y dos riegos de auxilio para complementar la aportación de las lluvias. 


\section{Germoplasma}

Se evaluaron 32 genotipos de frijol de diversos orígenes, que se adaptan a las condiciones agroclimáticas del Altiplano de México (Cuadro 1). Este grupo de genotipos incluyó fuentes de caracteres específicos para el mejoramiento genético, de los cuales no se conocía su reacción a los patógenos inductores de pudriciones de la raíz.

\section{Evaluaciones}

Las plantas de la primera fecha se evaluaron a los 33,39 y 46 días después de la siembra (dds), que co- rrespondieron a las etapas fenológicas $\mathrm{V}_{3}, \mathrm{R}_{5}$ y $\mathrm{R}_{6}$ (CIAT, 1987). En cada evaluación se extrajeron con ayuda de una pala, de cinco a diez plantas completas por variedad, tratando de obtener la mayor porción del sistema radical. Este se observó para detectar la presencia de síntomas de pudrición. Los síntomas se compararon visualmente con una escala de severidad de uno a nueve (CIAT, 1987) (Cuadro 2). Las plantas de la segunda fecha de siembra se evaluaron empleando la metodología señalada, a los 27, 34 y 49 dds, que también corresponden a las etapas fenológicas menciona-

Cuadro 1. Genotipos de frijol evaluados por su reacción a Fusarium spp. en el Campo Experimental Valle de México, P-V 1997.

\begin{tabular}{|c|c|c|c|}
\hline Genotipo & $\begin{array}{c}\text { Hábito de } \\
\text { crecimiento }\end{array}$ & Estatus biológico & Origen \\
\hline \multicolumn{4}{|l|}{ RAZA NUEVA GRANADA* } \\
\hline Bola 60 días & I & Criollo & Perú \\
\hline ICA Palmar (G 4523) & I & Mejorado & ICA, Colombia \\
\hline Michigan Dark Red Kidney & I & Mejorado & E.U. \\
\hline Perry Marrow & II & Mejorado & E. U. \\
\hline Kaboon & II & Mejorado & \\
\hline A 193 & II & Mejorado & CIAT, Colombia \\
\hline Canario 107 & I & Mejorado & INIFAP, México \\
\hline Cacahuate 72 & I & Mejorado & INIFAP, México \\
\hline Bayomex & I & Mejorado & INIFAP, México \\
\hline EPR-1-20 & I & Mejorado & INIFAP, México \\
\hline \multicolumn{4}{|l|}{ RAZA DURANGO } \\
\hline Pinto Villa & III & Mejorado & INIFAP, México \\
\hline Pinto Zapata & III & Mejorado & INIFAP, México \\
\hline BY 91023 & III & Mejorado & INIFAP, México \\
\hline Bayo Criollo del Llano & III & Criollo & Aguascalientes, México \\
\hline Bayo Baranda & III & Criollo & Zacatecas, México \\
\hline Chihuahua 86 & III & Criollo & Chihuahua, México \\
\hline MAM 48 & III & Mejorado & CIAT, Colombia \\
\hline \multicolumn{4}{|l|}{ RAZA JALISCO } \\
\hline G 2333 & IV & Criollo & Chiapas, México \\
\hline G 13746 & III & Criollo & México \\
\hline Garbancillo Zarco & IV & Criollo & Jalisco, México \\
\hline Amarillo de Calpan & III & Criollo & Puebla, México \\
\hline Puebla 152 & III & Criollo & Puebla, México \\
\hline México 332 & III & Criollo & México, México \\
\hline Flor de Mayo Bajío & III & Mejorado & INIFAP, México \\
\hline Flor de Mayo M38 & III & Mejorado & INIFAP/ CIAT \\
\hline FM 94006 & III & Mejorado & INIFAP, México \\
\hline Bayo Mecentral & III & Mejorado & INIFAP, México \\
\hline \multicolumn{4}{|l|}{ RAZA MESOAMERICANA } \\
\hline TLP 19 & II & Mejorado & Costa Rica/ CIAT \\
\hline TLP 20 & II & Mejorado & Costa Rica/ CIAT \\
\hline Negro Perla & I & Mejorado & INIFAP, México \\
\hline Negro Tacaná & II & Mejorado & INIFAP/ CIAT \\
\hline Negro INIFAP & II & Mejorado & INIFAP, México \\
\hline
\end{tabular}


Cuadro 2. Escala de severidad para evaluar pudriciones de raíz (Centro Internacional de Agricultura Tropical, 1987)

\begin{tabular}{cl}
\hline Grado de severidad & Apariencia del daño \\
\hline 1 & $\begin{array}{l}\text { sin síntomas visibles de la enfermedad } \\
\text { decoloración ligera, sin lesiones necróticas o con } 10 \% \text { de los tejidos del hipocótilo y de la raíz } \\
\text { cubiertos con lesiones } \\
\text { aproximadamente } 25 \% \text { de los tejidos del hipocótilo y de la raíz están cubiertos con lesiones, con } \\
\text { decoloración fuerte, aunque los tejidos estén firmes } \\
\text { aproximadamente } 50 \% \text { de los tejidos del hipocótilo y de la raíz están cubiertos con lesiones que se } \\
\text { combinan con ablandamiento, pudrición y reducción considerable del sistema radical } \\
\text { aproximadamente } 75 \% \text { o más de los tejidos del hipocótilo y de la raíz están afectados por estados } \\
\text { avanzados de pudrición, en combinación con una reducción severa del sistema radical. }\end{array}$ \\
\hline
\end{tabular}

das. Además, se calculó la incidencia (I ) de la pudrición de raíces por Fusarium spp., utilizando la fórmula propuesta por James (1974), donde I = número de plantas afectadas, expresadas como porcentaje del número total de plantas. De muestras de plantas infectadas tomadas al azar, se realizaron aislamientos en PDA, para corroborar que los inductores de los síntomas observados eran especies de Fusarium.

\section{Análisis de datos}

Con los datos de las pudriciones se realizaron análisis de varianza por fecha de siembra y de muestreo, bajo un diseño completamente al azar. La calificación de cada una de las plantas evaluadas se consideró como una repetición.

\section{Experimento 2}

Este experimento se estableció en un sitio próximo al experimento 1. Se sembraron ocho genotipos de frijol después del establecimiento de las lluvias el 17 de junio de 1997, en dos surcos de tres metros, con una distancia entre surcos de $60 \mathrm{~cm}$ y una densidad de 25 plantas $/ \mathrm{m}^{2}$. El diseño experimental fue factorial en arreglo de parcelas divididas, en el que la parcela grande fueron los tratamientos químicos y la pequeña los genotipos, con cuatro repeticiones.

\section{Germoplasma}

Se evaluaron ocho genotipos de frijol de diversos orígenes que se adaptan a las condiciones agroclimáticas del Altiplano de México, para los cuales se conocía su respuesta a la actividad de Fusarium spp. (Cuadro 4).

\section{Evaluaciones}

El porcentaje de emergencia se determinó a los 17 dds y la evaluación de la severidad de infección se realizó a los 19, 35, 49 y 73 dds, que corresponden a las etapas fenológicas $\mathrm{V}_{3}, \mathrm{R}_{5}, \mathrm{R}_{6}$ y $\mathrm{R}_{8}$ (CIAT, 1987). En cada evaluación se extrajeron del mismo surco cuatro plantas completas por variedad y por tratamiento, pro- curando obtener la mayor porción del sistema radical, el cual se observó para detectar la presencia de síntomas de pudrición, en raíz o cuello. Los síntomas se compararon visualmente con la escala de severidad ya mencionada (CIAT, 1987); además, se calculó la incidencia de pudrición de raíces por Fusarium spp. y $R$. solani, de acuerdo al método señalado (James, 1974). Se determinó el rendimiento en $\mathrm{kg} / \mathrm{ha}$ para todos los tratamientos. En el laboratorio se realizaron aislamientos en PDA para corroborar que los inductores de los síntomas observados eran Fusarium spp. y R. solani.

\section{Tratamientos}

Los fungicidas Captan 50\% (cis N-(triclorometiltio)-4 ciclohexeno-1,2 dicarboximida) y Benomyl 50\% (Metil-1-butil carbamoil-2-bencimidazol carbamato) se aplicaron a la semilla ( $2 \mathrm{~g} / \mathrm{kg}$ de semilla), de cada uno de los genotipos de frijol antes de la siembra.

\section{Análisis de datos}

Con los datos de las pudriciones se realizaron análisis de varianza utilizando un diseño factorial.

\section{RESULTADOS Y DISCUSIÓN}

\section{Evaluación de genotipos al ataque por Fusarium spp. (Experimento1).}

La severidad de la enfermedad se incrementó conforme avanzó el ciclo de cultivo y no se observaron genotipos inmunes (Cuadro 3). Las pudriciones de raíz fueron causadas principalmente por Fusarium solani f. sp. phaseoli, por F. oxysporum f. sp. phaseoli, y en baja frecuencia se observaron síntomas inducidos por Rhizoctonia solani. Los análisis de varianza para severidad de Fusarium spp. mostraron diferencias significativas $(\mathrm{P}<0,05)$ entre los genotipos para todas las evaluaciones, en ambas fechas de siembra. 
Cuadro 3. Reacción a Fusarium spp. de genotipos de frijol cultivados en el Campo Experimental Valle de México, Texcoco, Edo. de México, 1997.

\begin{tabular}{|c|c|c|c|c|c|c|}
\hline \multirow[b]{3}{*}{ Genotipo/Raza * } & \multicolumn{6}{|c|}{ Días después de la siembra (dds) } \\
\hline & \multicolumn{3}{|c|}{ Siembra 24/IV } & \multicolumn{3}{|c|}{ Siembra 8/V } \\
\hline & 33 & 39 & 46 & 27 & 34 & 49 \\
\hline \multicolumn{7}{|l|}{ RAZA NUEVA GRANADA } \\
\hline Bola 60 días & $5,5 * *$ & 5,9 & 8,8 & 3,8 & 4,6 & 4,8 \\
\hline ICA Palmar & 5,8 & 5,8 & 8,8 & 4,3 & 6,4 & 6,2 \\
\hline Michigan Dark Red Kidney & 5,6 & 6,2 & 9,0 & 4,5 & 6,2 & 6,4 \\
\hline Perry Marrow & 6,3 & 6,0 & 8,8 & 5,3 & 6,0 & 6,2 \\
\hline Kaboon & 6,6 & 5,9 & 9,0 & 5,1 & 6,2 & 6,0 \\
\hline A 193 & 5,3 & 5,3 & 7,8 & 3,3 & 4,0 & 6,6 \\
\hline Canario 107 & 6,0 & 6,2 & 8,2 & 4,4 & 4,2 & 5,8 \\
\hline Cacahuate 72 & 5,0 & 5,7 & 7,8 & 3,3 & 6,4 & 5,0 \\
\hline Bayomex & 5,0 & 6,0 & 8,6 & 2,8 & 3,0 & 6,8 \\
\hline EPR-1-20 & 6,0 & 6,1 & 9,0 & 5,0 & 3,8 & 5,6 \\
\hline \multicolumn{7}{|l|}{ RAZA DURANGO } \\
\hline Pinto Villa & 3,2 & 3,7 & 4,4 & 2,7 & 5,0 & 4,0 \\
\hline Pinto Zapata & 4,1 & 5,5 & 8,0 & 4,6 & 6,8 & 7,6 \\
\hline BY 91023 & 4,7 & 5,0 & 8,0 & 4,2 & 5,0 & 6,2 \\
\hline Bayo Criollo del Llano & 5,4 & 5,7 & 7,6 & 4,8 & 4,8 & 4,4 \\
\hline Bayo Baranda & 5,15 & 5,3 & 8,0 & 4,2 & 4,4 & 5,0 \\
\hline Chihuahua 86 & 4,7 & 4,7 & 8,6 & 4,6 & 5,8 & 6,4 \\
\hline MAM 48 & 4,5 & 6,0 & 9,0 & 4,7 & 5,4 & 7,8 \\
\hline \multicolumn{7}{|l|}{ RAZA JALISCO } \\
\hline G 2333 & 4,3 & 5,8 & 7,0 & 3,6 & 5,0 & 4,8 \\
\hline G 13746 & 4,6 & 5,9 & 7,4 & 7,1 & 6,6 & 6,8 \\
\hline Garbancillo Zarco & 4,5 & 5,3 & 8,0 & 5,3 & 5,0 & 5,2 \\
\hline Amarillo de Calpan & 3,2 & 4,8 & 7,8 & 3,4 & 1,6 & 3,2 \\
\hline Puebla 152 & 5,0 & 6,0 & 8,4 & 5,1 & 6,0 & 6,4 \\
\hline México 332 & 4,5 & 6,5 & 7,8 & 4,3 & 5,2 & 5,4 \\
\hline Flor de Mayo Bajío & 5,3 & 5,7 & 8,4 & 5,3 & 5,0 & 5,6 \\
\hline Flor de Mayo M 38 & 4,8 & 5,8 & 8,8 & 4,5 & 5,6 & 5,6 \\
\hline FM 94006 & 5,0 & 5,6 & 8,4 & 4,6 & 5,2 & 5,2 \\
\hline Bayo Mecentral & 5,1 & 5,5 & 7,6 & 5,2 & 3,8 & 6,2 \\
\hline \multicolumn{7}{|l|}{ RAZA MESOAMERICANA } \\
\hline TLP 19 & 5,4 & 4,8 & 8,2 & 4,4 & 5,8 & 7,8 \\
\hline TLP 20 & 3,5 & 4,2 & 7,0 & 4,3 & 4,8 & 7,0 \\
\hline Negro Perla & 2,7 & 4,1 & 6,0 & 5,6 & 5,0 & 4,6 \\
\hline Negro Tacaná & 3,9 & 5,8 & 7,8 & 5,1 & 3,6 & 3,2 \\
\hline Negro INIFAP & 5,9 & 4,8 & 8,2 & 4,3 & 5,4 & 5,4 \\
\hline DMS $* * *$ & 1,346 & 1,056 & 1,182 & 1,098 & 1,592 & 1,844 \\
\hline
\end{tabular}

* Singh, et al. 1991.

** Media de 5 a 10 repeticiones por evaluación. Descripción de la escala en el Cuadro 2.

$* * *$ DMS $(<\mathrm{P} 0,05)$

En la primera evaluación de la primera fecha de siembra, la amplitud de la severidad fue de tres a siete, los genotipos de origen andino de la raza Nueva Granada: Canario 107, G 4523, MDRK, Kaboon, EPR-120 y Perry Marrow, junto con la variedad Negro INIFAP (raza Mesoamericana) y el genotipo G 13746 (raza Jalisco) fueron los que mostraron el máximo grado de severidad. Los genotipos sobresalientes fueron: Negro Perla, Pinto Villa, Amarillo de Calpan y TLP 20, que pertenecen a diferentes razas. En la segunda evaluación la amplitud de la severidad fue de cuatro a seis, en donde los genotipos: Pinto Villa (raza Durango), Negro Perla y TLP 20 (raza Mesoamericana) mostraron una reacción intermedia. Mientras que en la tercera evaluación la amplitud de la severidad fue de cuatro a nueve; Pinto Villa fue la única variedad que mantuvo la calificación intermedia y el resto de los genotipos fueron susceptibles al ataque por Fusarium spp. (Cuadro 3, Fig. 1). 
La incidencia observada en las evaluaciones de la primera fecha de siembra fue del 80 al $100 \%$ (datos no presentados), lo que confirma la uniformidad de la infestación por Fusarium spp. en el suelo del sitio experimental, uniformidad tal vez favorecida por la siembra constante de frijol a través de los años y por la aplicación de riegos en el presente experimento.

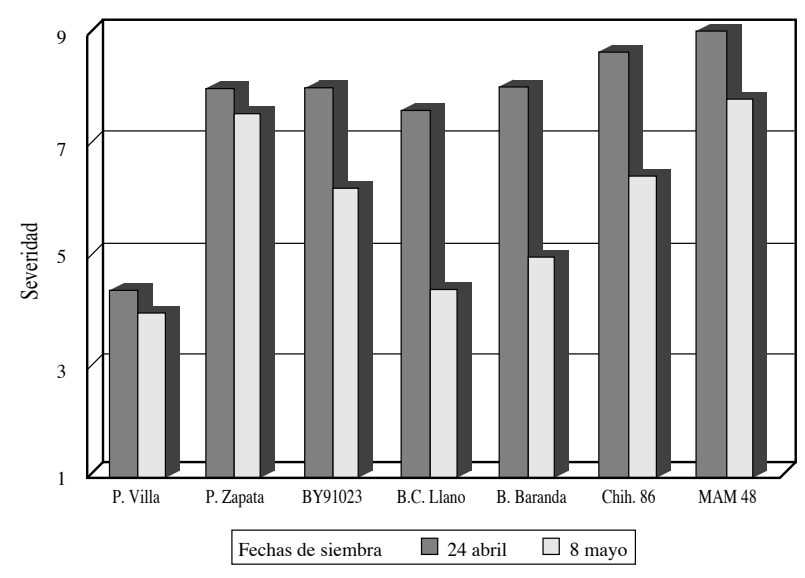

Figura 1. Reacción a Fusarium spp. de genotipos de frijol de la raza Durango, cultivados en el CEVAMEX.
En la segunda fecha de siembra en la primera evaluación, la amplitud de la severidad también fue de tres a siete, las variedades con mayor susceptibilidad fueron G 13746 y Negro Perla, las cuales pertenecen a diferentes acervos genéticos (Cuadro 4). Por otro lado, en la segunda evaluación la amplitud de la severidad fue de dos a siete, en ésta la variedad Amarillo de Calpan fue resistente y los genotipos Pinto Zapata y G 13746 resultaron con mayor susceptibilidad. En la tercera evaluación la amplitud de la severidad fue de tres a ocho, las mejores variedades fueron: Negro Tacaná, Amarillo de Calpan, Pinto Villa y Bayo Baranda. La variedad más resistente durante las tres evaluaciones en esta fecha de siembra fue Amarillo de Calpan, que se comportó como resistente al ataque por Fusarium spp., mientras que la variedad que presentó la mayor severidad (siete), desde la primera evaluación, fue G 13746, ambos genotipos pertenecen a la raza Jalisco (Cuadro 4).

La incidencia observada en la primera evaluación de la segunda fecha de siembra fue del 40 al 100\% (datos no presentados) y fue diferente entre variedades. Esta variación en la incidencia probablemente se debió a cambios en los factores climáticos no favorables para el desarrollo de la enfermedad y sólo se observó el 100\% de incidencia en los genotipos más susceptibles.

Cuadro 4. Reacción a Fusarium spp. y R. solani de ocho genotipos de frijol cultivados en un suelo naturalmente infestado, con y sin tratamiento químico a la semilla.

\begin{tabular}{|c|c|c|c|c|c|c|c|c|c|c|c|c|}
\hline \multirow[b]{3}{*}{ Genotipo/patógeno } & \multicolumn{12}{|c|}{ Días después de la siembra } \\
\hline & \multicolumn{4}{|c|}{ Testigo } & \multicolumn{4}{|c|}{ Captan * } & \multicolumn{4}{|c|}{ Benomyl * } \\
\hline & 19 & 35 & 49 & 73 & 19 & 35 & 49 & 73 & 19 & 35 & 49 & 73 \\
\hline Fusarium spp. & & & & & & & & & & & & Villa \\
\hline $1,0(* *)$ & 6,0 & 7,5 & 1,0 & 8,2 & 6,2 & 7,2 & 1,0 & 3,5 & 2,7 & 4,2 & 1,0 & \\
\hline Flor de Mayo Bajío & 2,5 & 1,5 & 7,0 & 1,0 & 6,0 & 6,7 & 6,7 & 1,0 & 4,2 & 3,5 & 6,5 & 1,0 \\
\hline Puebla 152 & 4,5 & 5,7 & 6,7 & 1,0 & 4,5 & 3,5 & 5,2 & 1,0 & 1,5 & 8,0 & 7,2 & 1,0 \\
\hline Negro 8025 & 3,7 & 5,7 & 7,0 & 1,0 & 3,7 & 1,7 & 7,2 & 1,0 & 4,2 & 6,7 & 6,0 & 1,0 \\
\hline Negro Tacana & 4,0 & 1,0 & 2,2 & 1,0 & 5,5 & 4,5 & 3,5 & 1,0 & 5,0 & 1,0 & 4,0 & 1,0 \\
\hline Negro Durango & 3,2 & 1,5 & 7,7 & 1,0 & 5,5 & 5,2 & 4,7 & 2,0 & 2,2 & 5,2 & 5,2 & 1,0 \\
\hline Negro Cotaxtla 91 & 4,7 & 6,2 & 5,5 & 1,0 & 4,0 & 2,7 & 5,5 & 1,0 & 5,2 & 4,7 & 5,5 & 1,0 \\
\hline BAT 477 & 2,0 & 1,5 & 4,2 & 1,0 & 2,0 & 4,5 & 4,2 & 2,0 & 1,5 & 2,7 & 5,7 & 1,0 \\
\hline \multicolumn{13}{|l|}{ R. solani } \\
\hline Pinto Villa & 5,0 & 6,0 & 1,0 & 6,2 & 1,0 & 5,0 & 1,0 & 4,7 & 1,0 & 5,5 & 1,0 & 6,5 \\
\hline Flor de Mayo Bajío & 2,7 & 4,5 & 1,0 & 5,7 & 1,0 & 1,0 & 1,0 & 8,2 & 2,0 & 4,7 & 1,0 & 6,2 \\
\hline Puebla 152 & 1,7 & 3,2 & 1,0 & 6,5 & 1,7 & 4,0 & 1,0 & 4,5 & 4,7 & 5,7 & 1,0 & 7,2 \\
\hline Negro 8025 & 1,7 & 5,2 & 1,0 & 7,0 & 1,0 & 3,2 & 1,0 & 6,0 & 3,0 & 2,5 & 1,0 & 7,0 \\
\hline Negro Tacana & 1,0 & 6,2 & 2,2 & 7,7 & 1,5 & 4,2 & 1,0 & 6,2 & 2,0 & 5,7 & 1,0 & 4,2 \\
\hline Negro Durango & 4,0 & 6,7 & 1,0 & 7,5 & 2,0 & 5,0 & 1,0 & 5,2 & 4,2 & 6,5 & 1,0 & 6,0 \\
\hline Negro Cotaxtla 91 & 1,0 & 7,0 & 1,0 & 7,2 & 1,0 & 7,2 & 1,0 & 4,5 & 2,2 & 6,7 & 1,0 & 6,2 \\
\hline BAT 477 & 2,2 & 4,2 & 1,0 & 6,2 & 1,0 & 5,2 & 3,0 & 4,5 & 1,2 & 3,5 & 1,0 & 5,2 \\
\hline
\end{tabular}

* aplicado a la semilla antes de la siembra ( $2 \mathrm{~g}$ de producto/kg de semilla).

** media de cuatro repeticiones por genotipo. Descripción de la escala en el Cuadro 2. 
En ninguno de los muestreos se observaron genotipos resistentes o inmunes a Fusarium spp., la mayoría mostraron valores de severidad mayores en la primera fecha de siembra, como se ejemplifica con la reacción de los genotipos de la raza Durango (Fig. 1). Solamente los genotipos Pinto Villa (intermedio) y Pinto Zapata (susceptible) (Fig. 1) y TLP 19, TLP 20 (susceptibles) tuvieron valores de severidad similares en la última evaluación en ambas fechas de siembra (Cuadro 3). Resultados similares en cuanto a la baja resistencia a las pudriciones de raíz, se observaron con anterioridad en el Altiplano semiárido (Acosta et al., 1991). Por otra parte, los genotipos de la raza Nueva Granada fueron los que mostraron el promedio de severidad más alto en ambas fechas de siembra (Fig. 2).

La variación en el grado de severidad observada en los genotipos entre y dentro de las fechas, indica por un lado la complejidad de la resistencia y por otro, sugiere la falta de homogeneidad genética de los genotipos, sobre todo de las variedades criollas. El inóculo existente en el sitio experimental resultó muy agresivo para los genotipos de origen andino. Campos (1987) señaló que las variedades de hábito determinado generalmente son más susceptibles que los de guía y semiguía a las pudriciones de raíz inducidas por Fusarium spp.; en esta investigación sólo se observó esta tendencia en las primeras lecturas, ya que al final hubo valores de severidad altos en ambos hábitos de crecimiento: determinado e indeterminado. Las enfermedades radicales son un problema de amplia distribución en México (López, 1991) y uno de los probables mecanismos de tolerancia es la emisión de raíces adventicias, sobre todo en genotipos de hábito indeterminado (tipo III) (Acosta et al., 1991; O’Brien et al., 1991).

Pastor-Corrales y Abawi (1987) indicaron que las variedades Garbancillo Zarco, Bayo Criollo del Llano y Puebla 152 tuvieron calificación intermedia al ser inoculados con $F$. oxysporum f. sp. phaseoli en invernadero y la variedad Cacahuate 72 se consideró resistente; a diferencia de la respuesta que mostraron en el presente trabajo, en que fueron susceptibles en la primera fecha de siembra y tuvieron reacción intermedia en la segunda fecha de siembra. El hecho de que los genotipos Cacahuate 72 y Negro 8025 resultaran resistentes a la inoculación con un aislamiento de Brasil (Pastor-Corrales y Abawi, 1987) y susceptibles en esta investigación, sugiere la presencia de una alta infestación en nuestro sitio de evaluación o la existencia de diferentes razas de Fusarium en cada uno de esos sitios de evaluación.

Además de la falta de resistencia en los genotipos, otros factores pueden facilitar la entrada de los hongos inductores de las pudriciones de la raíz, como son: el daño por otros patógenos y plagas, los daños por fertilizantes e insecticidas, el manejo inadecuado del cultivo con siembras profundas, los suelos apretados, la sequía y el pH desfavorable (Abawi et al., 1985). La combinación del control genético y cultural de las pudriciones inducidas por Fusarium spp. es una práctica recomendada para la producción de frijol ejotero en el oeste de los Estados Unidos (Silbernagel y Mills, 1990). Control que debería implementarse en el Altiplano de México, para disminuir los daños en el cultivo del frijol.

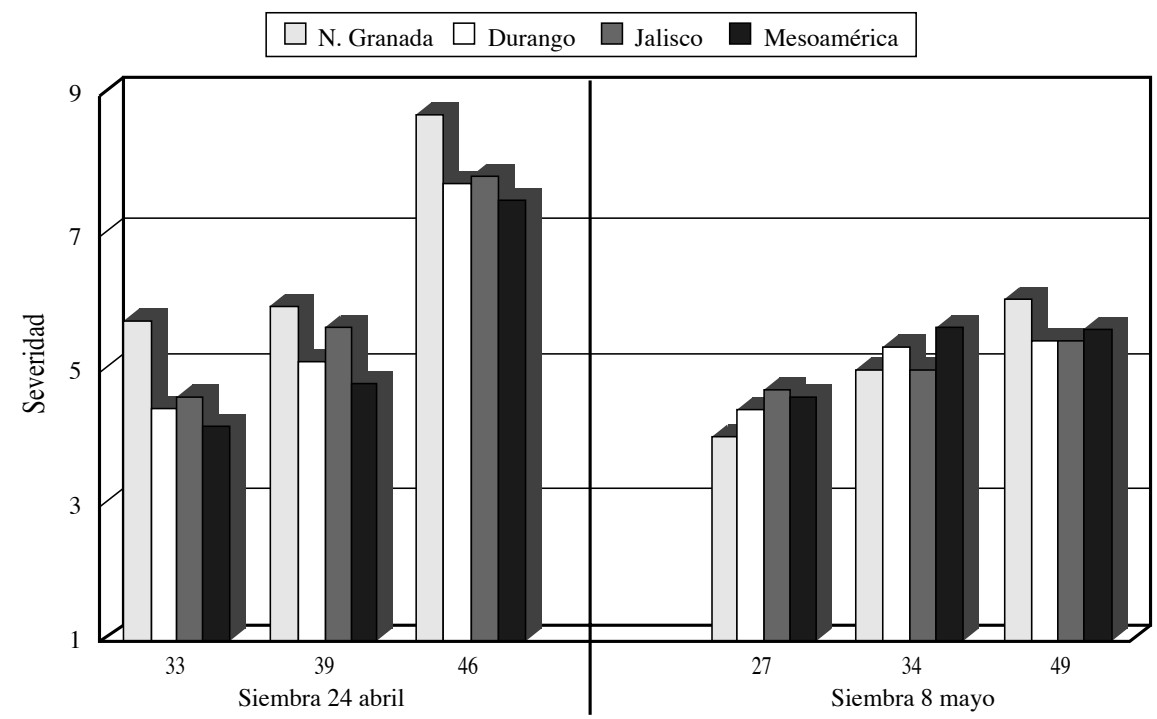

Figura 2. Pudriciones de raíz inducidas por Fusarium spp. en 32 genotipos de frijol de diferentes acervo genético. 
En contraste con las observaciones de Pastor-Corrales y Abawi, (1987) en relación a la lentitud de la infección y a la distribución de las pudriciones en manchones en el campo, en esta investigación la infección de las plantas en el campo fue rápida, con distribución uniforme y el daño por Fusarium spp. se observó tanto en raíces laterales como en la raíz principal y en el ta1lo. Estos resultados son similares a los que se obtuvieron en garbanzo en parcelas infestadas ex profeso para realizar evaluaciones de genotipos y conocer su reacción a Fusarium (López, 1974; Rheenen et al., 1992), de ahí la importancia de contar con parcelas uniformemente infestadas que permitan la evaluación de genotipos en el campo.

\section{Eficiencia del tratamiento a la semilla con} fungicidas para el control de Fusarium spp. y $\boldsymbol{R}$. solani, en un suelo naturalmente infestado. (Experimento 2).

La densidad de semillas empleada de 25 plan$\mathrm{tas} / \mathrm{m}^{2}$, fue adecuada para favorecer el desarrollo de la enfermedad y para obtener información confiable, debido a que los muestreos realizados implicaron la destrucción de las plantas. En general, la germinación y el establecimiento de las plántulas se vieron favorecidas por la aplicación de los fungicidas a la semilla, sobre todo en el caso de la aplicación de captan (Fig. 3). Estos resultados son similares a los señalados por Abawi y Pastor-Corrales (1990), quienes observaron que los fungicidas aplicados a la semilla generalmente protegen a las plantas por dos a tres semanas después de la siembra. En esta investigación la acción de los fungicidas (captan y benomyl) fue efectiva en la mayoría de los genotipos contra el ataque por Fusarium spp. duran- te siete semanas y en el caso de $R$. solani por diez semanas. Ambos productos se han señalado como fungicidas apropiados para el control de Fusarium spp. y de $R$. solani (Abawi y Pastor-Corrales, 1990).

En forma similar al experimento anterior, la severidad de la enfermedad se incrementó conforme avanzó el ciclo biológico del frijol (Cuadro 4) y la severidad de $F u$ sarium spp. alcanzó los máximos valores a los 49 dds.

En el tratamiento testigo la variedad Negro Tacaná alcanzó la mayor severidad a los 19 dds y las demás variedades lo hicieron a los $49 \mathrm{dds}$, pero a los $73 \mathrm{dds}$ no se observaron síntomas de Fusarium spp. (Cuadro 4). La severidad de $R$. solani fue alta en las variedades Pinto Villa y Negro Durango desde la primera evaluación (19 dds) y la mayoría de las variedades alcanzaron la máxima severidad a los 35 dds. A los 49 dds no se observaron síntomas de $R$. solani en ninguno de los genotipos, pero si de Fusarium spp. y a los 73 dds la situación fue a la inversa (Cuadro 4).

En el tratamiento con captan la variedad Pinto Villa mostró susceptibilidad a Fusarium spp. desde la primera evaluación. Las variedades Pinto Villa, Flor de Mayo Bajío y Negro 8025 tuvieron los valores más altos de severidad a los 49 dds, mientras que Negro Cotaxtla 91 la presentó a los 35 dds (Cuadro 4). Por otro lado, BAT 477 obtuvo los menores valores de severidad durante todas las evaluaciones. En cuanto a la respuesta a $\mathrm{R}$. solani las variedades Flor de Mayo Bajío, Negro 8025 y Negro Tacaná fueron protegidas durante 73 dds y en esta evaluación Flor de Mayo Bajío mostró el mayor valor de severidad (Cuadro 4).

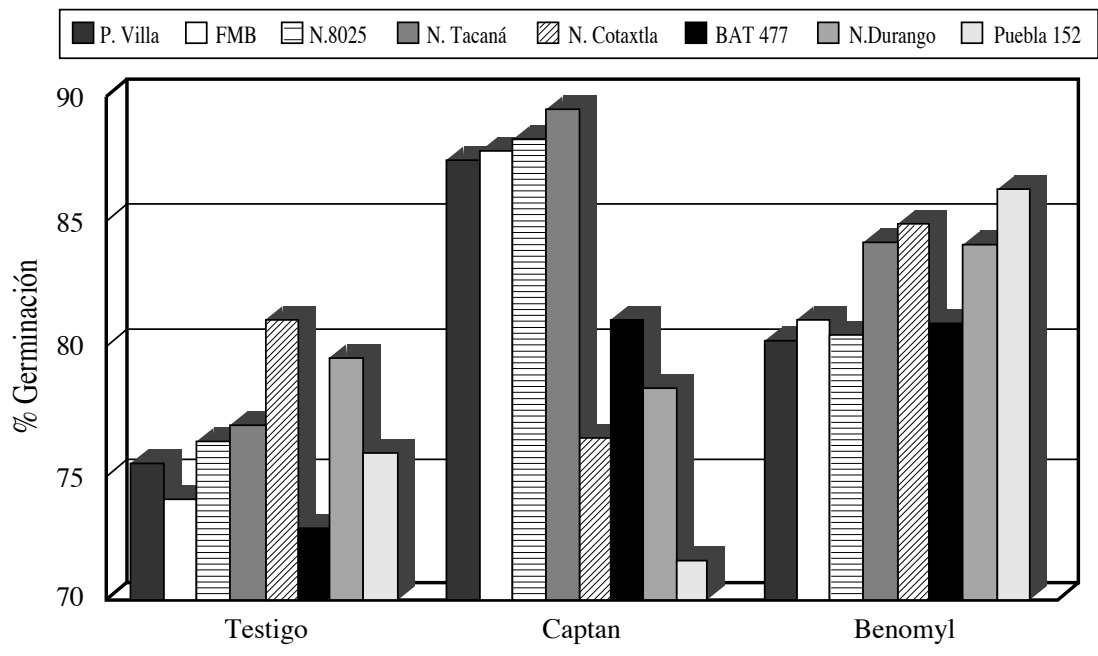

Figura 3. Germinación de semilla de frijol, con y sin tratamiento químico, sembrada en suelo infestado con Fusarium spp. y R. solani. 
En el tratamiento con benomyl la variedad Negro Cotaxtla 91 mostró una severidad de cinco desde los 19 dds y la variedad Pinto Villa tuvo el menor nivel de severidad a Fusarium spp. en todas las evaluaciones. No se observó un patrón de protección para la mayoría de las variedades. Puebla 152 y Negro 8025 alcanzaron altos niveles de susceptibilidad a los 35 dds y fueron los que mostraron la mayor susceptibilidad a los 49. A los 73 dds no se observaron síntomas de Fusarium spp. (Cuadro 4). La infección por $R$. solani tuvo el máximo valor de severidad a los 35 dds; no se observaron síntomas a los 49 dds y la severidad se incrementó a los 73 dds. Las variedades BAT 477 y Negro 8025 fueron los genotipos que mostraron los menores valores de severidad hasta la evaluación de los 73 dds, en que alcanzaron los valores 5 y 7 , respectivamente (Cuadro 4).

En relación con el rendimiento, la mayoría de las variedades mostraron incrementos en los tratamientos con protección, en un rango de 1,4 a 33,0\%, excepto las variedades Negro Tacaná, Negro Durango y BAT 477, que tuvieron reducciones en el rendimiento entre 1,0 y 62,9\% (Cuadro 5). Aún cuando los valores de severidad por el ataque de Fusarium spp. y R. solani fueron de reacción intermedia, es importante señalar que durante el ciclo biológico de las variedades se observó la incidencia de enfermedades foliares como roya (Uromyces appendiculatus var. appendiculatus), antracnosis (Colletotrichum lindemuthianum), tizón común (Xanthomonas campestris pv. phaseoli) y tizón de halo (Pseudomonas syringae pv. phaseolicola). En el caso de las variedades mencionadas, las plantas de los tratamientos con captan y benomyl, productos que no controlan a los patógenos inductores de las enfermedades mencionadas, presentaron niveles altos de severidad de tizón común y

Cuadro 5. Rendimiento en $\mathrm{kg} / \mathrm{ha}$ de ocho genotipos de frijol cultivados en un suelo naturalmente infestado con Fusarium spp. y R. solani, con y sin tratamiento a la semilla.

\begin{tabular}{|c|c|c|c|}
\hline Genotipo & Testigo & Captan * & Benomyl * \\
\hline Pinto Villa & 1,644 & $1,898(15,4) * *$ & $2,188(33,1)$ \\
\hline Flor de Mayo Bajío & 1,939 & $2,148(10,8)$ & $2,228(14,9)$ \\
\hline Puebla 152 & 2,072 & $2,460(18,7)$ & $1,563(-32,6)$ \\
\hline Negro 8025 & 2,031 & $2,326(14,5)$ & $2,159(6,3)$ \\
\hline Negro Tacana & 1,829 & $1,794(-1,9)$ & $1,398(-30,8)$ \\
\hline Negro Durango & 2,512 & $1,545(-62,9)$ & $2,396(-4,8)$ \\
\hline Negro Cotaxtla 91 & 2,261 & $2,292(1,4)$ & $2,321(2,6)$ \\
\hline BAT 477 & 2,095 & $1,597(-31,2)$ & $1,983(-5,6)$ \\
\hline Promedio & 2,048 & $2,007(-2,0)$ & $2,029(-1,0)$ \\
\hline
\end{tabular}

* Aplicado a la semilla antes de la siembra, en una dosis de dos gramos de producto/kg de semilla.

** Porcentaje de incremento o decremento. roya a los 78dds, en comparación con las plantas del tratamiento testigo (datos no presentados), lo cual pudo haber influido en la disminución del rendimiento.

Durante el ciclo del cultivo se observó una fuerte competencia entre los hongos inductores de pudriciones de raíz para colonizar las plantas de frijol (Cuadro $4)$. $R$. solani se considera un buen competidor y colonizador de materia orgánica, sobre todo cuando hay buenas condiciones de humedad en el suelo (Abawi, comunicación personal, 1991). En este caso, aparentemente se produjo una sucesión ecológica en el desarrollo de estos hongos conforme avanzó el desarrollo del cultivo en el tiempo (Cuadro 4).

\section{CONCLUSIONES}

La incidencia de Fusarium spp. en la primera fecha de siembra fue de $80 \%$ en la primera evaluación y de $100 \%$ en las siguientes, lo que confirma la uniformidad de la infestación del suelo. La severidad de la pudrición se incrementó conforme avanzó el ciclo de cultivo en el grupo de materiales evaluados y no se observaron altos niveles de resistencia a Fusarium spp., ya que la mayoría de los genotipos resultaron susceptibles a Fusarium spp., sobre todo a los 46 dds $\left(\mathrm{R}_{6}\right)$.

El tratamiento químico a la semilla influyó en la germinación, en el establecimiento de las plántulas, en la protección a las plantas contra las pudriciones de la raíz y se observó una interacción genotipo-tratamiento. La variedad BAT 477 obtuvo los menores valores de severidad contra Fusarium spp tratada con captan y Pinto Villa con benomyl.

Se observó competencia entre Fusarium spp. y $R$. solani por colonizar a las plantas de frijol, lo que realizaron como sucesión ecológica.

Las plantas de variedades tolerantes, afectadas por Fusarium spp. emitieron raíces adventicias, lo que les permitió tener rendimientos aceptables.

\section{LITERATURA CITADA}

ABAWI, G. S.; CROSIER, D. C.; COBB, A. C. 1985. Root rot of snap beans in New York. New York's Food and Life Sciences Bulletin Num. 110:1-7.

ABAWI, G. S. 1989. Root rots. In: Schwartz, H. F., and M. A. Pastor-Corrales (eds.). Bean production problems in the tropics. 2nd Ed. Cali, Colombia. CIAT (Centro Internacional de AgriculturaTropical) pp.105-158. 
ABAWI, G. S.; PASTOR, M. A.C. 1990. Root rots of beans in Latin America and Africa: Diagnosis, research methodologies, and management strategies. Cali, Colombia. Centro Internacional de Agricultura Tropical (CIAT). $114 \mathrm{p}$.

ACOSTA, G. J. A.; NAVARRETE, M. R. 1996.Identificación de genotipos de frijol con resistencia múltiple a enfermedades en la Mesa Central de México. Agric. Téc. México. 22 :187-197.

ACOSTA, G. J. A; NAVARRETE, M. R.; OCHOA, M. R. 1991. Identificación de material tolerante a las pudriciones de raíz en frijol de temporal. Phaseolus, Pub. Especial No. 8. INIFAP-MSU. Durango, México. pp. 103-108.

BECERRA, N.; LÓPEZ, S. E. 1994. Enfermedades del cultivo del frijol en el trópico de México. XXI Congreso Nacional de Fitopatología. Soc. Mex. de Fitopatología. Cuernavaca, Mor. p. 56.

CAMPOS, A. J. 1987. Enfermedades del Frijol. México. Trillas. $132 \mathrm{p}$.

CENTRO INTERNACIONAL DE AGRICULTURA TROPICAL (CIAT). 1987. Sistema estándar para la evaluación de germoplasma de frijol. Schoonhoven A. v. y M.A. Pastor-Corrales (comps.). Cali, Colombia. Centro Internacional de Agricultura Tropical $52 \mathrm{p}$.

ESTEVEZ, DE J. C.; MERONUCK, R.; PERCICH, J. A. 1998. Etiology and control of kidney bean root rot in Minnesota. Ann. Rep. Bean Improv. Coop. 41:55-57.

GARCÍA, E. 1981. Modificaciones al sistema de clasificaciones climáticas de Köeppen. UNAM. México. 252 p.

JAMES, W. C. 1974. Disease assessment keys. Ann. Rev. Phytopath. 12:27-48.

LÓPEZ, F. L. C. 1991. Definición de prioridades de investigación fitopatológica para la zona templada del Altiplano Central de México. Agric. Téc. México. 17: 17-54

LÓPEZ, G. H. 1974. Herencia del carácter de resistencia a marchitez (Fusarium sp.) en garbanzo (Cicer arietinum) bajo condiciones de campo. Agric. Téc. México. 3:286-289.
NAVARRETE, M. R.; ACOSTA, G. J. A. 1996. Sanidad y germinación de semilla de frijol producida en diferentes fechas de siembra. Phaseolus. Publicación especial No. 8 INIFAP-MSU. Durango, México. pp. 169-178

O'BRIEN, R. G.; O'HARE, P. J.; GLASS, R. J. 1991. Cultural practices in the control of bean root rot. Australian J. Exp. Agric. 31:551-555.

PASTOR C.M. A.; ABAWI, G. S. 1987. Reactions of selected bean germ plams to infection by Fusarium oxysporum f. sp. phaseoli. Plant Dis. 71:990-993.

RODRÍGUEZ, G. R. 1996. Calidad física y sanitaria de la semilla de frijol utilizada por los agricultores de Durango para la siembra en temporal. Memorias del XXIII Congreso Nacional de la Sociedad Mexicana de Fitopatología. Guadalajara, Jal. p. 80.

RHEENEN, V. H. A.; REDDY, M. V.; KUMAR, J.; HAWARE, M. P. 1992. Breeding for resistance to soil-borne diseases in chickpea. In: Disease resistance breeding in chickpea. Singh, K. B., and C. Saxena (eds.) International Center for Agricultural Research in the Dry Areas (ICARDA). Aleppo, Syria. pp. 55-70.

SÁNCHEZ, A. J. H.; CÁRDENAS, M. 1988. Etiología y daño de las pudriciones radicales del frijol (Phaseolus vulgaris L.) en el estado de Durango. Rev. Chapingo 12(58-59): 43-49.

SILBERNAGEL, M. J.; MILLS, L. J. 1990. Genetic and cultural control of Fusarium root rot in bush snap beans. Plant Dis. 74:61-66.

SINGH, S. P. 1992. Common bean improvement in the tropics. Plant Breed. Rev. 10: 199-269.

SINGH, S. P.; GEPTS, P.; DEBOUCK, D. G. 1991. Races of common bean (Phaseolus vulgaris, Fabaceae). Econ Bot. 45: 379-396.

VELASQUEZ V. R.; SCHWARTZ, H. F. 1997. Reaction of selected mexican and USA bean lines to Fusarium oxysporum f. sp. phaseoli. Ann. Rep. Bean Improv. Coop. 40:95-96. 